\title{
Importancia de la detección de dientes supernumerarios con tomografía computada de haz cónico
}

\author{
Importance of the detection of supernumerary teeth with conic beam computed tomography
}

\section{Señor Editor:}

Los dientes supernumerarios (SN) son anomalías del desarrollo dental que pueden definirse como dientes que exceden en número a los habituales en la cavidad oral, estos pueden o no estar asociados a algún síndrome (Displasia Cleidocraneal); su prevalencia se encuentra entre $0.4 \%$ y $3 \%$, según diversos autores y se presenta con mayor frecuencia en la niñez, más en mujeres que en hombres (1).

Estos dientes (SN) pueden encontrarse en distintas regiones de los maxilares además distintas localizaciones entre ellas se encuentras los(mesiodens) presentes en la línea media siendo el más común de los casos; le siguen los premolares (parapremolares), los laterales y los molares (paramolar) $(2,3)$.

Los (SN) son de morfología variada pueden ser cónicos, barril o mora. Los dientes suplementarios (eumorficos) que se hallan en la dentición caduca y estos no tienen ninguna variedad en su anatomía a deferencia de los rudimentarios (dismorficos) que se presentan en la dentición permanente y tienen variedad anatómica $(4,5)$.

La localización adecuada de los dientes (SN) es muy importante para una correcta planificación del tratamiento y su diagnóstico. Tradicionalmente, se los diagnosticaban y localizaban mediante métodos radiográficos bidimensionales (2D), como vistas panorámicas, radiografías cefalométricas, periapicales u oclusales (6).

Con el fin de superar las deficiencias de la imagen en $2 \mathrm{D}$, se han incrementado significativamente las solicitudes de tomografía computada de haz cónico (TCHC), siendo éste el estudio imagenológico ideal para la evaluación de los dientes SN (7).

La detección temprana de los dientes (SN) puede ser muy ventajosa para evitar futuras complicaciones. El tratamiento depende de la posición exacta, tamaño y tipo de $\mathrm{SN}$, así como su repercusión sobre el diente temporal o permanente adyacente como reabsorción radicular externa, proximidad a estructuras anatómicas de importancia como el conducto dentario inferior, el conducto naso palatino, piso de las fosas nasales. La TCHC brinda toda esta información $(8,9)$.

Los reportes de casos y la literatura sugieren que la remoción de un diente $(\mathrm{SN})$ en la dentición primaria no es recomendable ya que puedes provocar el desplazamiento del diente permanente durante la operación y se menciona que muchos de los dientes SN temporales erupcionan gracias a la presencia de los espacios interdentales (10).

Kevin Roberto Romero Díaz 1,a;2,b, Ana Paola Trevejo Bocanegra 2,c, Vilma Elizabeth Ruiz García de Chacón 2,c

Universidad Estatal de Guayaquil. Guayaquil, Ecuador.

Facultad de Estomatología Roberto Beltrán, Universidad Peruana Cayetano Heredia. Lima, Perú.

Cirujano Dentista

Residente de la Especialidad de Radiología Bucal y Maxilofacial.

Especialista en Radiología Oral y Maxilofacial. 


\section{Correspondencia:}

Kevin Roberto Romero Díaz

Correo electrónico: kevin.romero@upch.pe

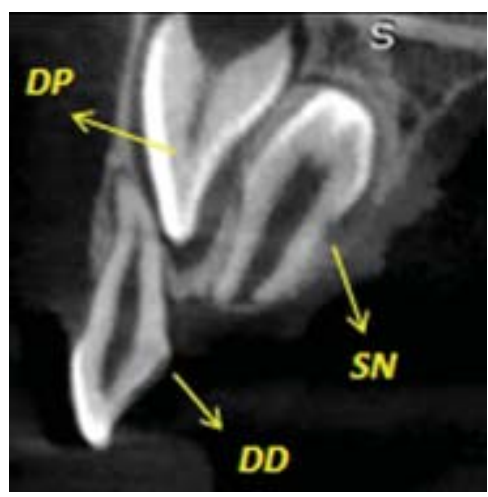

Figura 1.Ejemplo representativo de una tomografía computada de haz cónico (TCHC) que muestra en un corte sagital un diente supernumerario $S N$ en el maxilar superior en posición invertida que dificulta la correcta erupción del diente permanente DP, el diente deciduo DD no se encuentra afectado. Imagen tomada del archivo del Servicio de Radiología Bucal y Maxilofacial - Universidad Peruana Cayetano Heredia.

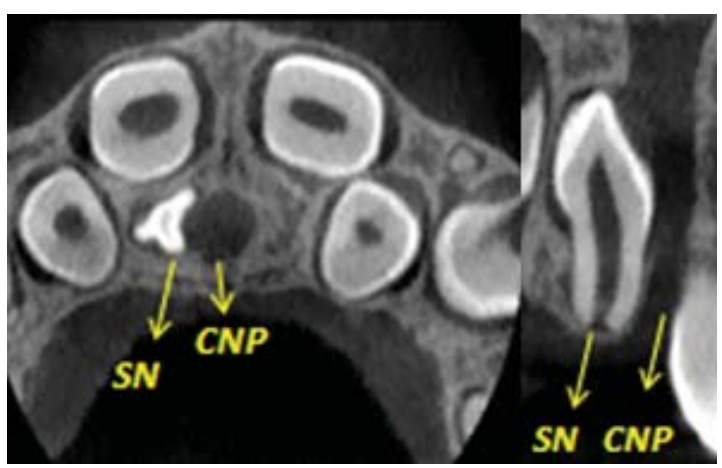

Figura 2. Ejemplo representativo de una tomografía computada de haz cónico (TCHC) en corte axial y coronal que muestra un diente supernumerario $S N$ en el maxilar superior en posición invertida adyacente al conducto naso palatino CNP. Imagen tomada del archivo del Servicio de Radiología Bucal y Maxilofacial - Universidad Peruana Cayetano Heredia.

\section{REFERENCIAS BIBLIOGRÁFICAS}

1. Demiriz L, Hazar Bodrumlu E, İçen M, Durmuşlar MC. Evaluation of the accuracy of cone beam computed tomography on measuring impacted supernumerary teeth. Scanning. 2016;38(6):579-84. doi: $10.1002 /$ sca. 21303

2. Sebastián $\mathrm{C}$, Izquierdo $\mathrm{B}$, Gutiérrez $\mathrm{C}$, Aso A. Dientes supernumerarios: claves esenciales para un adecuado informe radiológico. Rev Arg Radiol. 2016;80(4):258-67.

3. Sane VD, Chandan S, Patil S, Patil K. Cone Beam Computed Tomography heralding new vistas in appropriate diagnosis and efficient management of incidentally found impacted Mesiodens. J Craniofac Surg. 2017;28(2):e105-6.

4. Mossaz J, Kloukos D, Pandis N, Suter VGA, Katsaros C, Bornstein MM. Morphologic characteristics, location, and associated complications of maxillary and mandibular supernumerary teeth as evaluated using cone beam computed tomography. Eur J Orthod. 2014;36(6):708-18.

5. Ersin NK, Candan U, Alpoz AR, Akay C. Mesiodens in primary,mixed and permanent dentition a clinical and radiographic study. J Clin Pediatr 2004; 28 (4): 295-8

6. Albert A, Mupparapu M. Cone beam computed tomography review and classification of mesiodens: Report of a case in the nasal fossa and nasal septum. Quintessence Int (Berl). 2018;49(5):413-7.

7. Mason C, Azam N, Holt RD, Rule DC. A retrospective study of unerupted maxillary incisors associated with supernumerary teeth. Br J Oral Maxillofac Surg. 2000;38(1):62-5.

8. Ferrés-Padró E, Prats-Armengol J, Ferrés-Amat E. A descriptive study of 113 unerupted supernumerary teeth in 79 pediatric patients in Barcelona. Med Oral Patol Oral Cir Bucal. 2009;14(3):1-7.

9. Mossaz J, Kloukos D, Pandis N, Suter VGA, Katsaros C, Bornstein MM. Morphologic characteristics, location, and associated complications of maxillary and mandibular supernumerary teeth as evaluated using cone beam computed tomography. European J Orthod. 2014;36(6):708-18.

10. Jammal N, Silva R. Prevalencia de dientes supernumerarios en una muestra mexicana. Rev Mex Ortod. 2015;3(2):88-91.

Recibido: 20-03-2019

Aceptado: 12-04-2019 